\title{
Predictions of daily contagions of CoViD-19 from October 10, 2020 until February 2021 in Italy with a new Epidemiologic FRActal Model plus Expolinear (EpiFRAME) to better frame the pandemic due to the 2B-BetaSarbeco-virus SARS-CoV-2
}

Calogero Rinzivillo

Department of Medical-Surgical Sciences, Organs Transplantation and Advanced Technologies Policlinic of University of Catania, Italy

\section{Sergio Conte}

School of Advanced International Studies on Applied Theoretical and non Linear Methodologies of Physics, Bari, Italy

\section{Francesco Casciaro}

School of Advanced International Studies on Applied Theoretical and non Linear Methodologies of Physics, Bari, Italy

\section{Renato Bernardini}

University of Catania School of Medicine, Italy

Antonio Cascio

University of Palermo, Palermo, Italy

\section{Elena Benigni}

WONDER (World Organization for Noetic, Dimensional and Evolutionary Research), Rome, Italy

Ines Paola Monte

University of Catania, Italy

Ferda Kaleagasioglu

Istinye University İstanbul, Turkey

\section{Fang Wang}

Hunan Agricultural University, Changsha, China

\section{Cengiz Mordeniz}

Tekirdag Namik Kemal University, Turkey

\section{Giuseppe Liberti}

Cannizzaro Hospital, Catania, Italy

\section{Corrado Spatola}

University of Catania, Italy

Elio Conte ( $\sim$ elio.conte@fastwebnet.it) 
School of Advanced International Studies on Applied Theoretical and non Linear Methodologies of Physics, Bari, Italy

\section{Short Report}

Keywords: Covid-19, Fractal modelling, Prediction of contagions

Posted Date: November 4th, 2020

DOl: https://doi.org/10.21203/rs.3.rs-102042/v1

License: (c) (1) This work is licensed under a Creative Commons Attribution 4.0 International License. Read Full License 


\title{
Predictions of daily contagions of CoViD-19 from October 10, 2020 until February 2021 in Italy with a new Epidemiologic FRActal Model plus Expolinear (EpiFRAME) to better frame the pandemic due to the 2B-BetaSarbeco-virus SARS-CoV-2
}

\author{
Calogero Rinzivillo ${ }^{(1)}$, Sergio Conte ${ }^{(2)}$, Francesco Casciaro $^{(2,3)}$, Renato Bernardini ${ }^{(4)}$, Antonio \\ Cascio $^{(5)}$, Elena Benigni ${ }^{(6)}$, Ines Paola Monte ${ }^{(7)}$, Ferda Kaleagasioglu ${ }^{(2,8)}$, Fang Wang ${ }^{(9)}$, Cengiz \\ Mordeniz $^{(2,10)}$, Giuseppe Liberti ${ }^{(11)}$, Corrado Spatola ${ }^{(12)}$, Elio Conte ${ }^{(2)}$ \\ (1) Department of Medical-Surgical Sciences, Organs Transplantation and Advanced Technologies -Policlinic \\ of University of Catania, Italy. \\ (2) School of Advanced International Studies on Applied Theoretical and non Linear Methodologies of \\ Physics, Bari, Italy. \\ (3) U.O.C. "Medicine and Surgery of Urgency", "Sacro Cuore di Gesù" Hospital in Gallipoli (Le), Italy \\ (4) Department of Biomedical and Biotechnological Sciences, Section of Pharmacology, University of Catania \\ School of Medicine, Italy. \\ (5) Head of Infection Disease and Department of Health Promotion, Maternal and Infant Care, Internal \\ Medicine and Medical Specialties, University of Palermo, Palermo, Italy \\ ${ }^{(6)}$ WONDER (World Organization for Noetic, Dimensional and Evolutionary Research), Rome, Italy. \\ ${ }^{(7)}$ Department of General Surgery and Medical-Surgical Specialities- University of Catania, Italy \\ ${ }^{(8)}$ Department of Pharmacology and Clinical Pharmacology, Istinye University Topkapı Campus, Maltepe \\ Neighbourhood, Teyyareci Sami Street, Building no.3, Zeytinburnu, İstanbul, Turkey, 34010 \\ ${ }^{(9)}$ Hunan Agricultural University, Changsha, China \\ (10) Department of Anesthesiology, Intensive care and pain Medicine, Tekirdag Namik Kemal University, \\ Turkey \\ ${ }^{(11)}$ CoViD-19 Regional Commissioner - Division of Infective Diseases, Cannizzaro Hospital, Catania, Italy. \\ 12) Radio Oncology Unit of Policlinic and Department of Medical-Surgical Sciences, Organs Transplantation \\ and Advanced Technologies - University of Catania, Italy.
}

\begin{abstract}
We study a new fractal model for prediction of contagions of CoViD-19 using experimental data from October 10, 2020 to predict contagions from November until February 2021 in Italy. We obtain that the time of the peak is estimated to be at November 11, 2020 when number of contagions will be about 38489 cases.
\end{abstract}

\section{Introduction}

The aim of the present work is to effect a prediction of the contagions of SARS-CoV-2 in Italy using experimental data in the period from October 10, 2020 to February 2021.In the work we use the methods of the fractal analysis, fitting a new fractal model following the performance that was used previously from Ziff and Ziff [1] during the contagions in China. The current prediction, effected by such new model introduced by us, is that the size of the epidemic will be about 38489 cases of contagions in Italy and the time of peak will be about November 11, 2020 on the general plane, depending instead the actual size of 
the process from the respect or an increase o decrease of the prevention measures that are fixed from the governing bodies.

\section{Materials and Methods}

The power-law (fractal) behavior has been postulated and applied in epidemic studies of Corona Virus disease in China. It is related to the properties of the networks that carry out the propagation of the disease. Vazquez [2] developed a network model, Anna L. Ziff and Robert M. Ziff [1] applied a fractal behavior model in contagions in China. The daily number of new contagions cases, $n(t)$, in an epidemic followed a power-law with an exponential cutoff

$n(t)=k t^{\gamma} \exp \left(-t / t_{0}\right)$

This model was used in our previous estimations $[3,4,5]$. We have modified such model introducing an additive linear term $C(\mathrm{n})$. Therefore, the model that we adopt is the following

$n(t)=k t^{\gamma} \exp \left(-t / t_{0}\right)+C(n)$

where $C(n)$ is a linear parameter that is established by the fitting operation.

\section{Results}

We applied the previous model for the contagions of SARS-CoV-2 in Italy. The parameter values that we estimate are the follows

$k=2.1486$

$\gamma=3.8671$

$\mathrm{t}_{0}=8.4377$

$C(n)=6435.77$

Statistics of parameters $(r=0.98)$

\begin{tabular}{|c|c|c|}
\hline parameter & $\begin{array}{c}\text { standard } \\
\text { error }\end{array}$ & Range $(95 \%$ confidence $)$ \\
\hline $\mathrm{k}=2,1486$ & 0,8517 & $0,3944 \div 3,902$ \\
\hline$\gamma=3,8671$ & 0,1210 & $3,6057 \div 4,1046$ \\
\hline $\mathrm{t}_{0}=8,4377$ & 2,8860 & $2,7977 \div 14,0777$ \\
\hline$C(n)=6435,77$ & 657,9700 & $5080,64 \div 7790,90$ \\
\hline
\end{tabular}

They confirm that we are in presence of a fractal regime given by the non-integer value of $\gamma$. The value $\gamma \mathrm{t}_{0}$ represents the Time of the Peak. The results are in Figures 1, 2, 3. The experimental data explored is from October 10, 2020 to February 2021. It is seen that the time peak is about the November 11, 2020, with a total of contagions of about 38489 cases. 


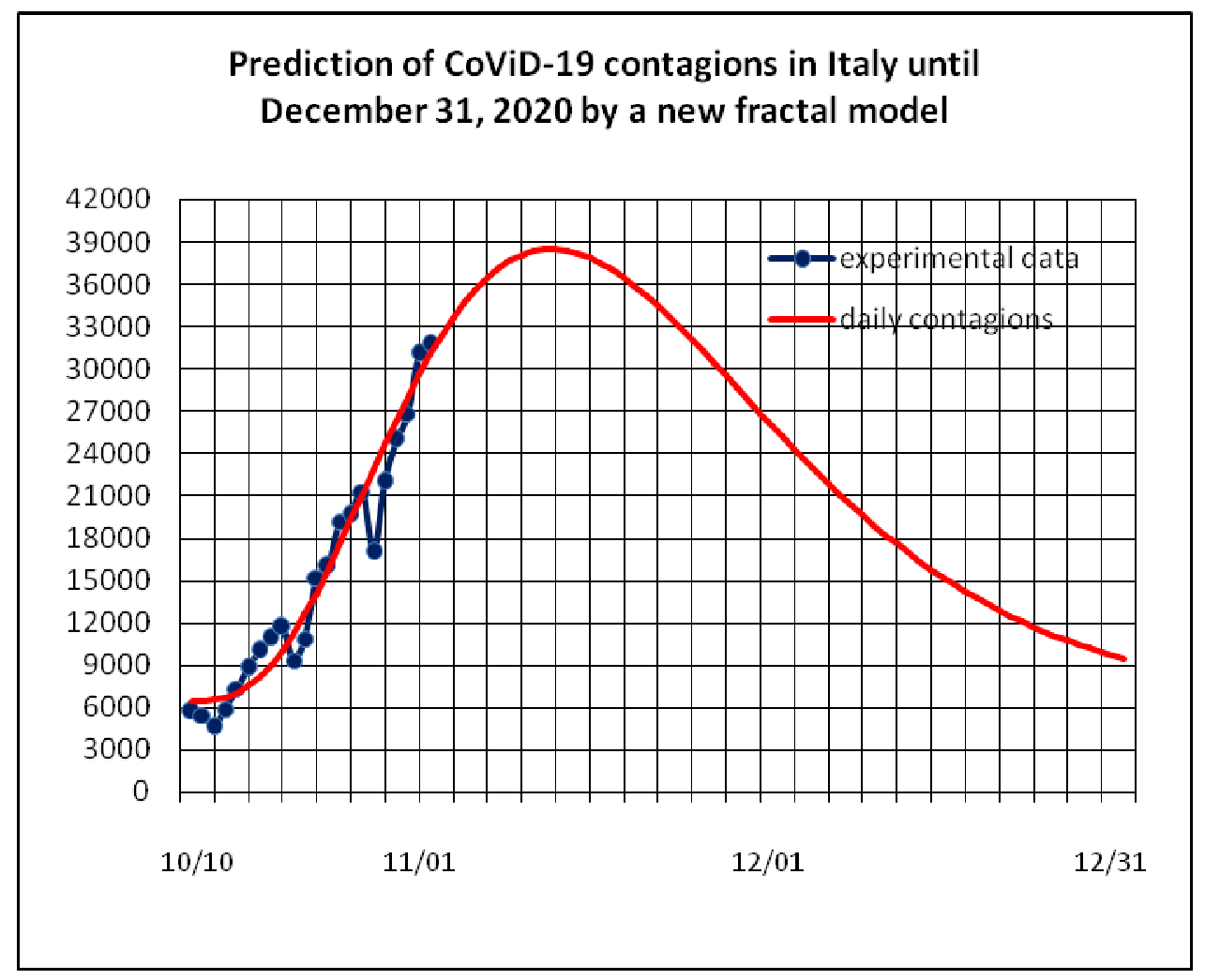

Figure 1

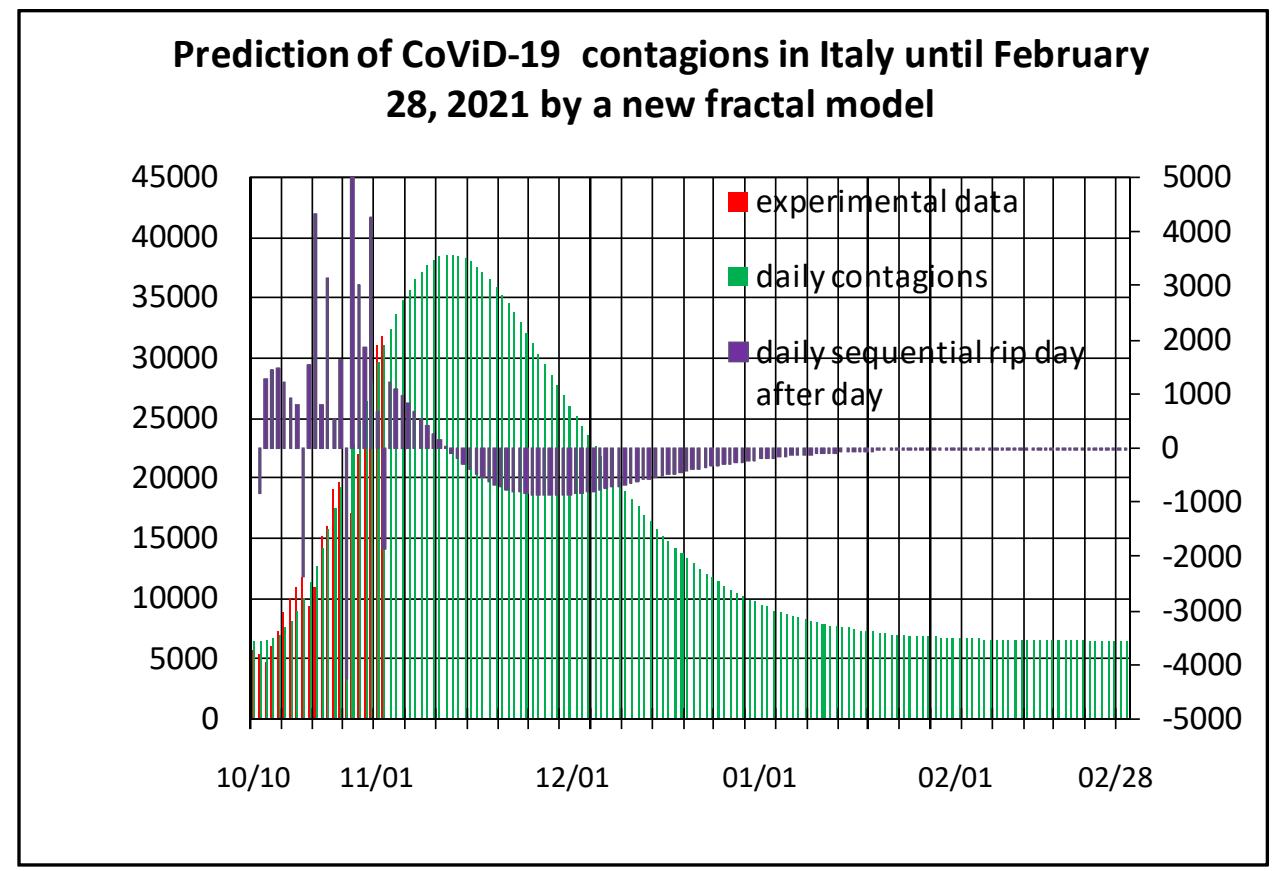

Figure 2 


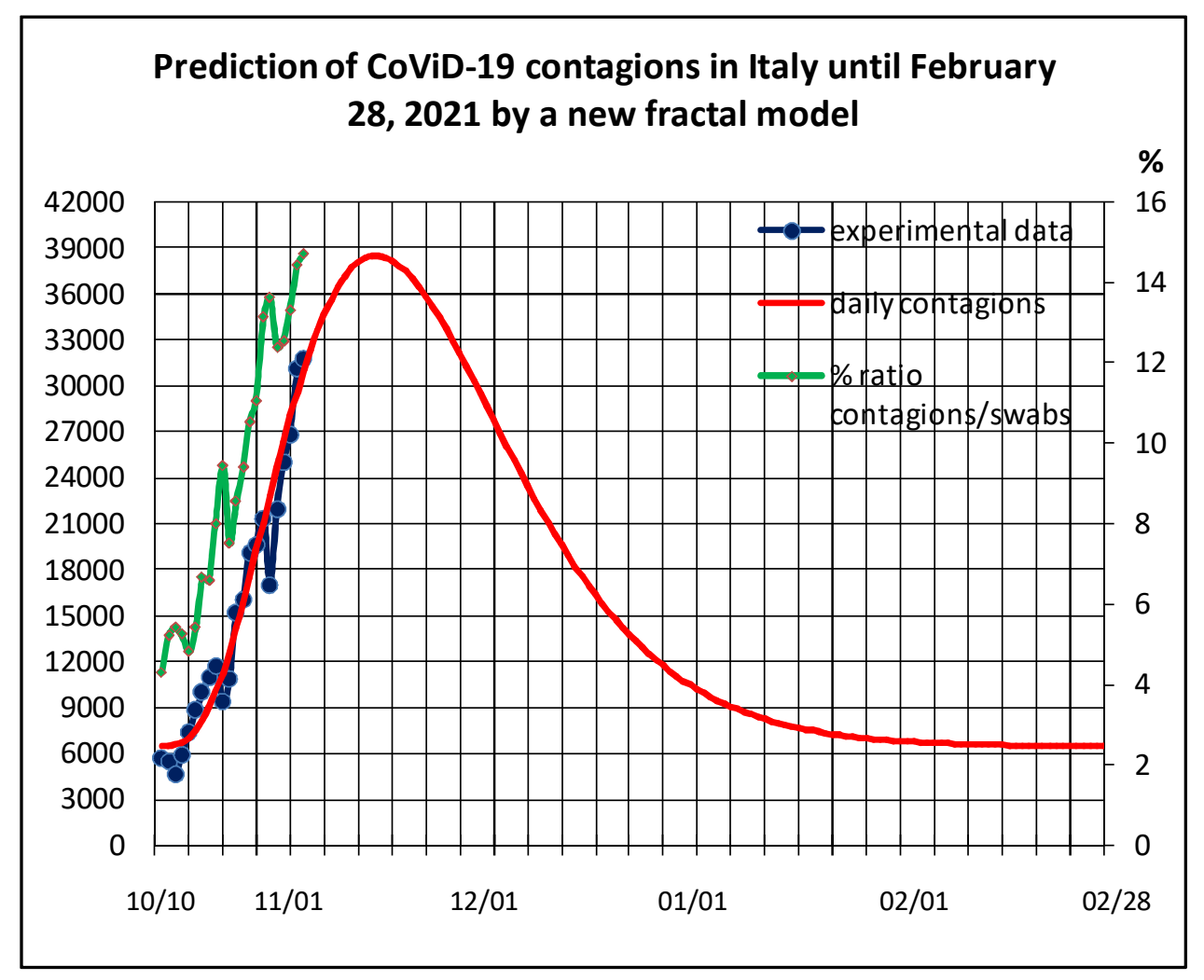

Figure 3

We calculated also the correlation existing daily between the number of contagions and the swabs made. The results that follow, indicate that such correlation exist at an high statistics level since the P-value is of < 0,0001 and the $P$ value summary has three stars.

Statistics
\begin{tabular}{|l|l|}
\hline Number of XY Pairs & 22 \\
\hline Pearson $r$ & 0,8515 \\
\hline $95 \%$ confidence interval & 0.6706 to 0.9368 \\
\hline P value (two-tailed) & $P<0,0001$ \\
\hline P value summary & $* * *$ \\
\hline Is the correlation significant? (alpha $=0.05)$ & Yes \\
\hline R squared & 0,7250 \\
\hline
\end{tabular}

\section{References}

[1] Anna L. Ziff and Robert M. Ziff, Fractal kinetics of COVID-19 pandemic (with update 3/1/20),medRxiv preprint doi: https://doi.org/10.1101/2020.02.16.20023820.

[2] Alexei Vazquez. Polynomial growth in age-dependent branching processes with diverging reproductive number. Physical Review Letters, 96(3):038702, January 2006. ISSN 0031-9007, 1079-7114.doi: 10.1103/ Phys. Rev. Lett.96.038702. URL http://arxiv.org/abs/cond-mat/0505116. arXiv: cond-mat/0505116. 
[3] Calogero Rinzivillo, Sergio Conte, Ferda Kaleagasioglu, Fang Wang, Alberto Foletti, Elio Conte, The Basic Indications and the Clinical Implications of Being the SARS Covid-19 a multifractal. vixra [v1] 2020-04-07

[4] Calogero Rinzivillo, Sergio Conte, Ferda Kaleagasioglu, Fang Wang, Alberto Foletti, Elio Conte, Prediction of Daily Contagions of Covid-19 from March 01 to May 19 in Italy. vixra[v1] 2020-03-17

[5] Calogero Rinzivillo, Sergio Conte, Ferda Kaleagasioglu, Fang Wang, Alberto Foletti, Cengiz Mordeniz, Elio Conte, New Prediction of Sars-Covid-19 in Italy. vixra [v1] 2020-04-24 
Figures

\section{Prediction of CoViD-19 contagions in Italy until December 31,2020 by a new fractal model}

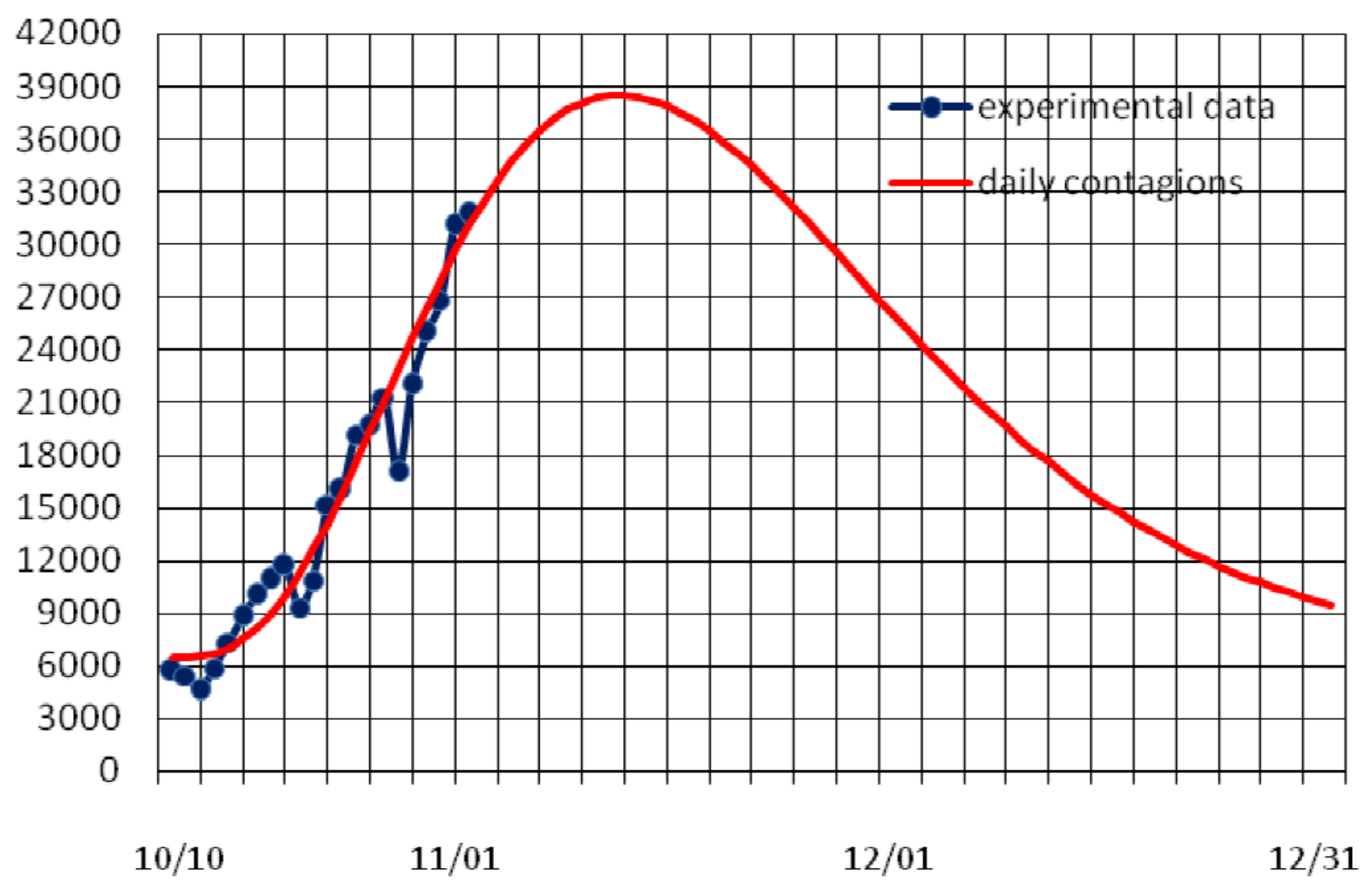

Figure 1

A caption was not provided with this version 


\section{Prediction of CoViD-19 contagions in Italy until February 28,2021 by a new fractal model}

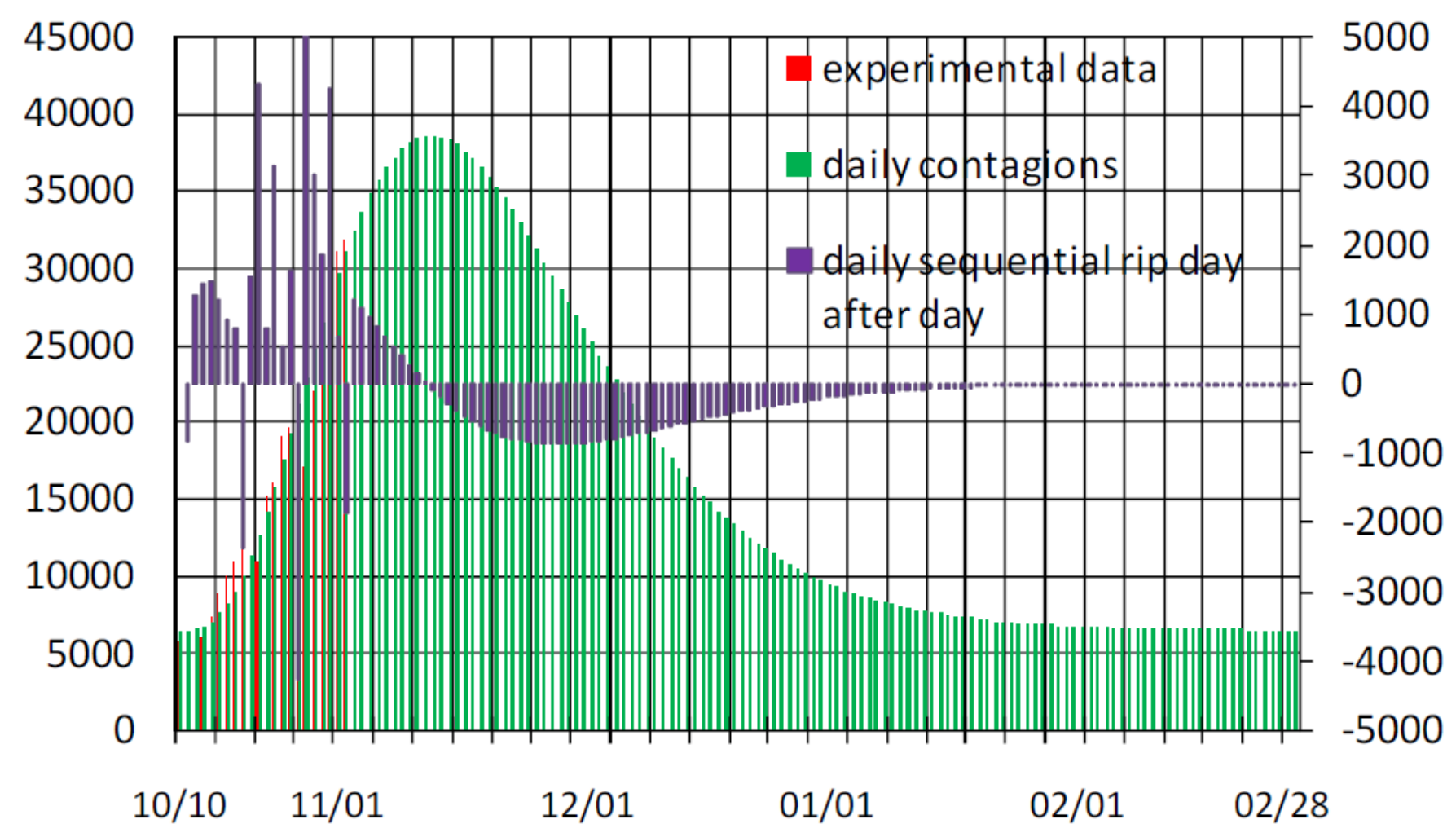

Figure 2

A caption was not provided with this version 


\section{Prediction of CoViD-19 contagions in Italy until February 28,2021 by a new fractal model}

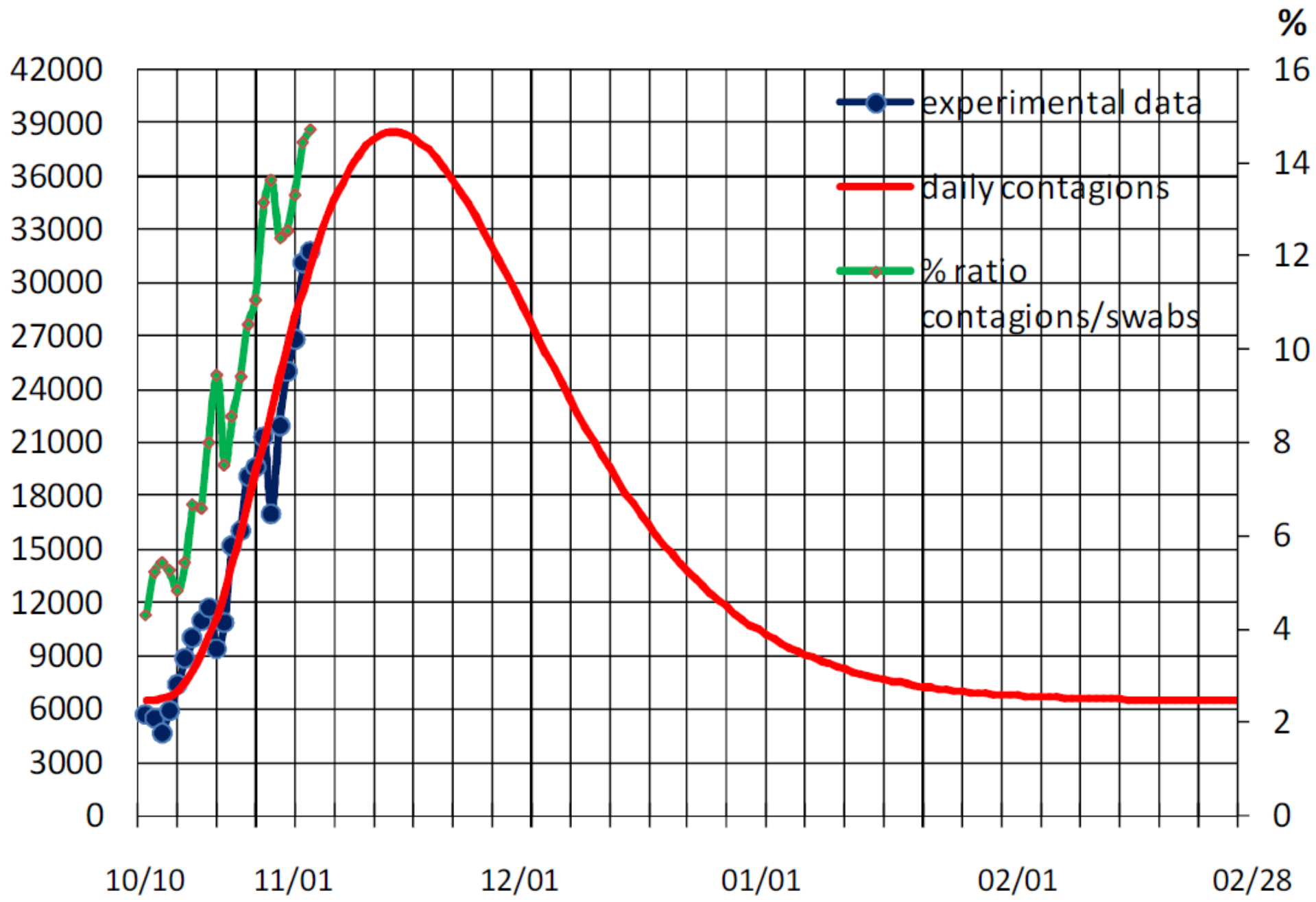

Figure 3

A caption was not provided with this version 